\title{
BEHAVIOR OF TPC'S IN A HIGH PARTICLE FLUX ENVIRONMENT *
}

A. Etkin, S.E. Eiseman, K.J. Foley, R.W. Hackenburg, R.S. Longacre, W.A. Love, T.W. Morris, E.D. Platner, A.C. Saulys Brookhaven National Laboratory

S.J. Lindenbaum BNL -47192 Brookhaven National Laboratory and City College of New York

C.S. Chan, M.A. Kramer, K.H. Zhao, Y. Zhu City College of New York

T.J. Hallman, L. Madansky Johns Hopkins University

S. Ahmad, B.E. Bonner, J.A. Buchanan, C.N. Chiou, J.M. Clement, G.S. Mutchler and J.B. Roberts, Rice University

\author{
Paper to be published in \\ Proceedings of the Symposium on RHIC Detector R\&D \\ Brookhaven National Laboratory \\ October 10-11, 1991
}

December 13, 1991

* This research was supported by the US Department of Energy under Contract Nos. DE-AC02-76CH00016, DE-AC02-83ER40107, DE-AS05-81ER40032 and DE-AC0276ER03274, and the City University of New York PSC-BHE Research Award Program. 


\title{
Behavior of TPC's in a High Particle Flux Environment
}

\author{
A. Etkin, S.E. Eiseman, K.J. Foley, R.W. Hackenburg, R.S. Longacre \\ W. A. Love, T.W. Morris, E.D. Platner and A.C. Saulys \\ Brookhaven National Laboratory, Upton, New York 11973 \\ S.J. Lindenbaum \\ Brookhaven National Laboratory and City College of New York \\ C.S. Chan, M.A. Kramer, K.H. Zhao and Y. Zhu \\ City College of New York, New York, New York 10091 \\ T.J. Hallman and L. Madansky \\ Johns Hopkins University, Baltimore, Maryland 21218
}

S. Ahmad, B.E. Bonner, J.A. Buchanan, C.N. Chiou, J.M Clement
G.S. Mutchler, and J.B. Roberts
Bonner Nuclear Laboratory, Rice University, Houston, Texas 77251

Abstract

TPC's (Time Projection Chamber) used in E-810 at the AGS (Alternating Gradient Synchrotron) were exposed to fluxes equivalent to more than $10^{7}$ minimum ionizing particles per second to find if such high fluxes cause gain changes or distortions of the electric field. Initial results of these and other tests are presented and the consequences for the RHIC (Relativistic Heavy Ion Collider) TPC-based experiments are discussed.

\section{Introduction}

In order to study the performance of TPC's in a high particle flux environment we have made a number of measurements utilizing the AGS experiment E-810 TPC's[1]. This experiment uses 3 TPC modules in a vertical dipole magnetic field (Fig. 1). Silicon and proton beams can be directed to a variety of nuclear targets ranging from $S i$ to $P b$. In the forward hemisphere the experiment has high acceptance and has reconstructed events with up to 130 charged particle tracks from a heavy target. Results of the initial analysis will be presented, distortions in the electric field due to positive ions, two vertex resolution and reconstruction efficiency at high track density. Application of these results to the question of luminosity limitation capability of the "STAR"[2] experiment at RHIC will be discussed.

\section{DISCLAIMER}

This report was prepared as an account of work sponsored by an agency of the United States Government. Neither the United States Government nor any agency thereof, nor any of their employees, makes any warranty, express or implied, or assumes any legal liability or responsibility for the accuracy, completeness, or usefulness of any information, apparatus, product, or process disclosed, or represents that its use would not infringe privately owned rights. Refercnce herein to any specific commercial product, process, or service by trade name, trademark, manufacturer, or otherwise does not necessarily constitute or imply its endorsement, recommendation, or favoring by the United States Government or any agency thereof. The views and opinions of authors expressed herein do not necessarily state or reflect those of the United States Government or any agency thereof. 


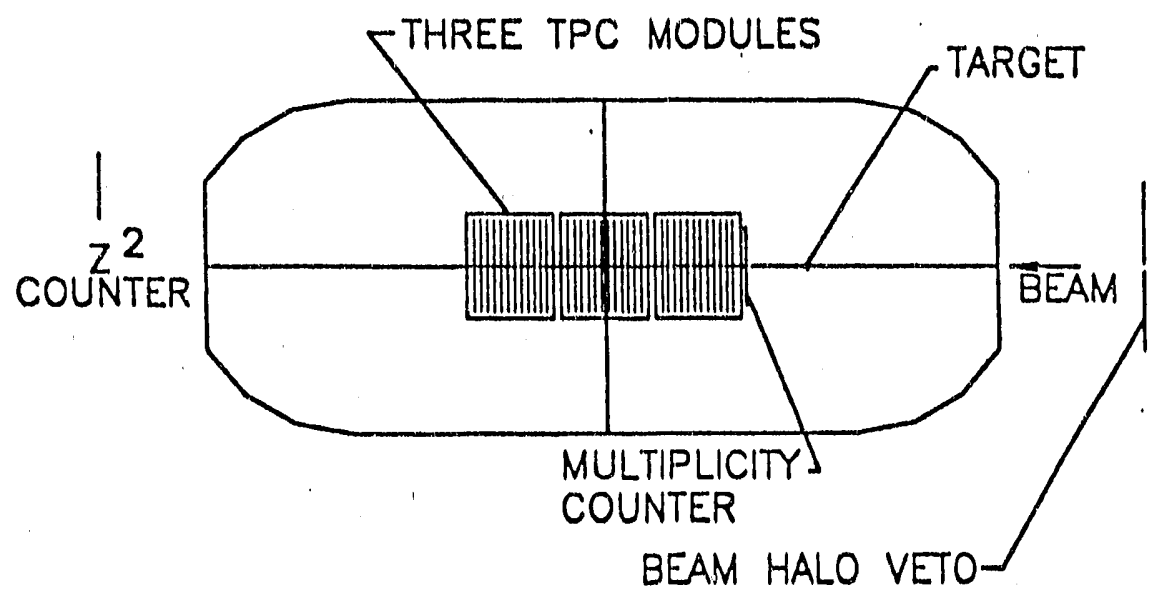

Figure 1: Plan view of E-810 apparatus.

\section{Distortions Due To Ion Loading}

Figure 2 shows a head on view of an E-810 TPC. The ion beam passes directly through the center of the TPC. The beam is typically $1.5 \mathrm{~cm}$ wide by $0.6 \mathrm{~cm}$ high in the center of the first TPC module. The AGS beam has a spill duration of 1 second. About $300 \mathrm{~ms}$ after the beam starts positive ions created at the beginning of the spill reach the negative HV electrode. At this point a sheet of ions has formed from the beam to the HV electrode. This produces a distortion of the "uniform" electric field. In fact the electrons which are drifting down will see a reduction in electric field near the beam causing the drift velocity to be reduced in the vicinity of the beam. We have studied this effect by raising the $S i$ beam to $67 \mathrm{~K}$ per 1 second spill. This then produces, after $\sim 300 \mathrm{~ms}$, a positive ion loading of $10^{7}$ ions $/ \mathrm{cm}^{3}$ along the bearn path. Two distortions are produced, the first is in the drift direction $Y$ and the second is in the horizontal direction $X$. In order to measure these effects the difference between the projected beam track position and the position determined in the TPC is calculated. Projected beam track positions are determined using two sets of $X$ and $Y$ measuring beam PWC's. A run at low rate ( $4 \mathrm{~K}$ ions per spill) with a defocussed bearn (10 cm wide by $12 \mathrm{~cm}$ high) is used to determine the relationship between row numbers, wire numbers and drift time, and the trajectory position in the TFC. The differences $\Delta X$ and $\Delta Y$ (projected position - TPC position) are calculated for subsequent runs. Figure 3 shows $\Delta Y$ in a high rate run at the center of the first TPC module as a function of event number during the spill with $30 \mathrm{~ms}$ between events. As can be seen, after $\sim 300 \mathrm{~ms}$ the distortion reaches a maximum and remains relatively constant until near the end of the spill when the beam intensity drops. This is consistent with the development of an ion sheet during this time that reaches equilibrium after $\sim 300 \mathrm{~ms}$. 


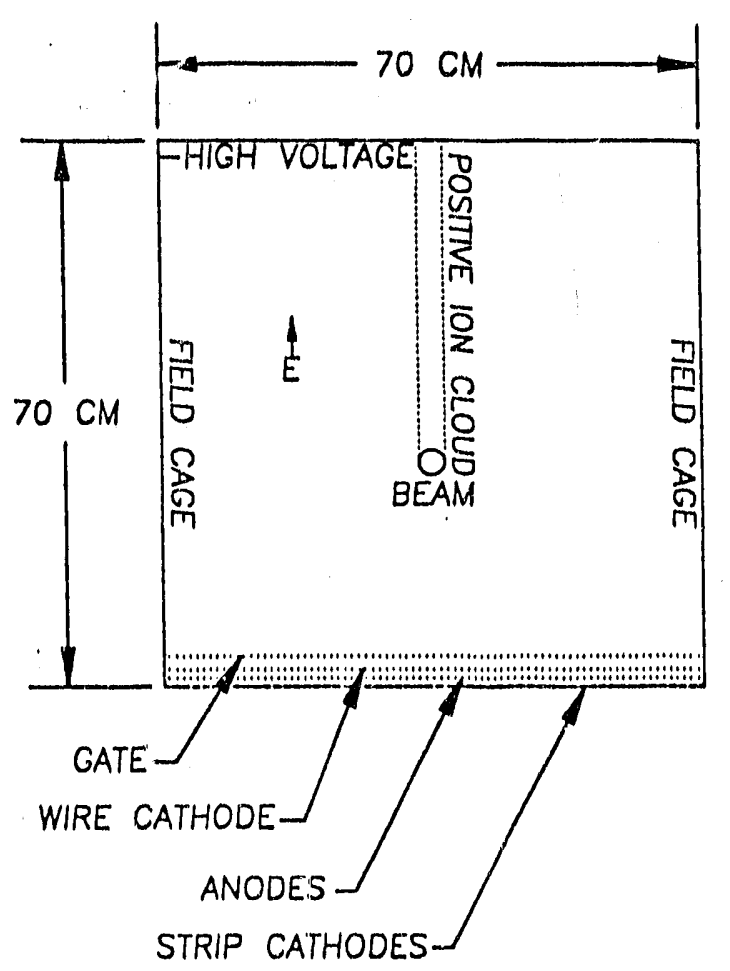

Figure 2: Section of a TPC looking along the beam showing beam spot and positive ion sheet.

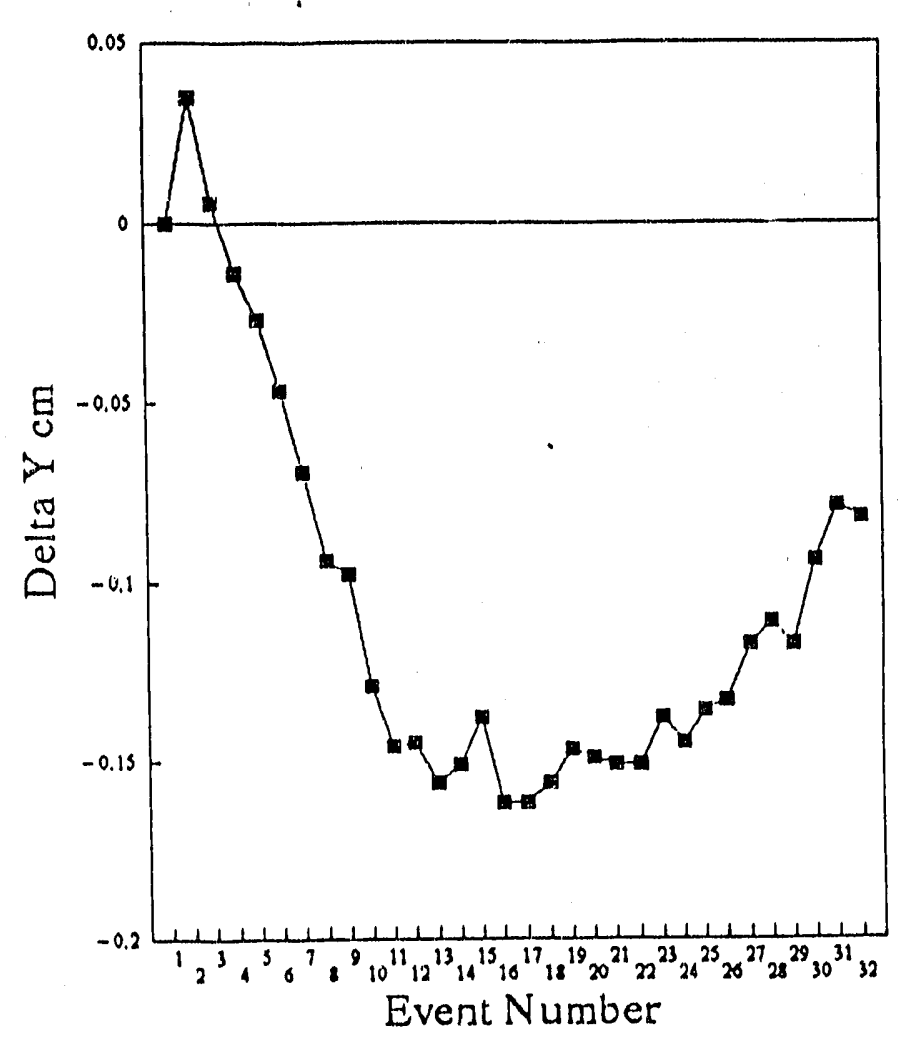

Figure 3: $\Delta Y$ versus spill event number for small beam.

Each TPC has 12 rows surrounded by a field cage. In Fig. 4 we plot the distortion, $\Delta Y$, versus row number (rows $19 \& 20$ were not working and are not plotted) after $360 \mathrm{~ms}$ into the spill for a small beam. We see the first and last rows (nearest to the field cage) have much less distortion then the middle. This appears to be because of the proximity of the field cage which minimizes the electric field distortions caused by the ion sheet. Figure 5 shows $\Delta Y$ versus row number measured using a large beam $(10 \mathrm{~cm} \times 12 \mathrm{~cm})$. In both cases $\Delta \mathrm{Y}^{\prime}$ is negative indicating a decrease in the effective drift velocity in agreement with preliminary electrostatic calculation which give a $2 \mathrm{~mm}$ maximum shift. Figure 6 shows the $\Delta X$ measured in the middle of the first TPC module, versus $X$ beam for the small $(1.5 \mathrm{~cm}$ by $0.6 \mathrm{~cm}$ ) beam. $\Delta X$ is largest near the edge of the beam and zero at the beam center as expected if the drifting electrons are attracted to the positive ion sheet.

At RHIC the Central TPC will have a minimum ionizing track rate of $2 \times 10^{6}$ tracks per second for $A u-A u$ collisions. At the inner radius this is spread out over an area of 3 $\mathrm{m}^{2}$ or 70 tracks per $\mathrm{cm}^{2}$ per second. The E-810 test had a track density of $>10^{7}$ minimum ionizing tracks in a $1 \mathrm{~cm}^{2}$ spot yet this ion loading caused a distortion in the drift direction of only $1.5 \mathrm{~mm}$. 


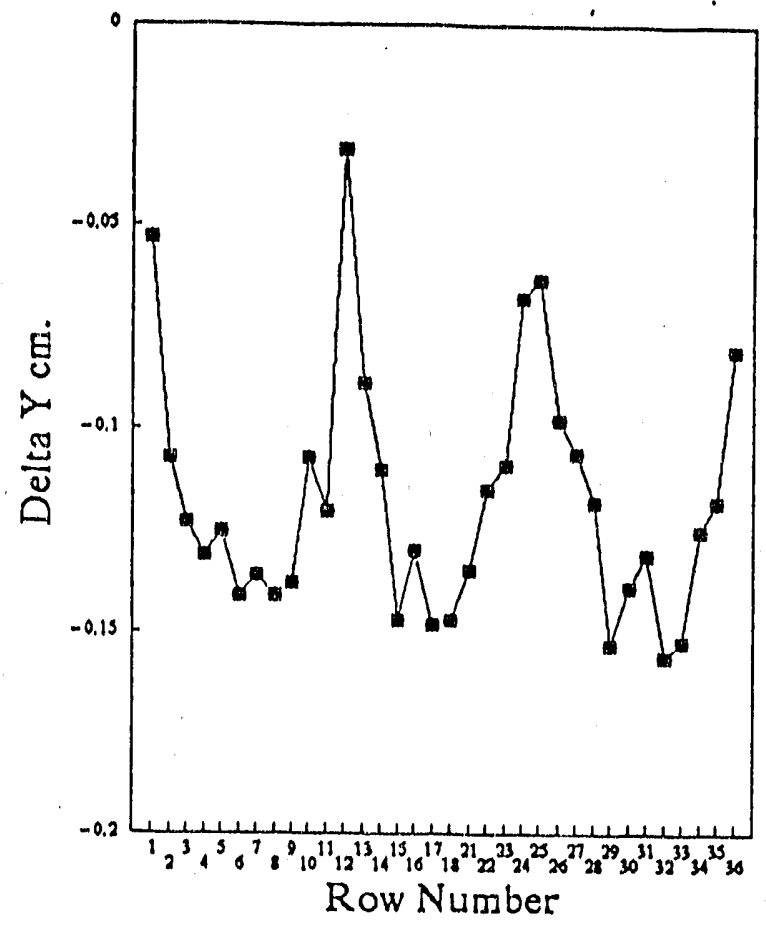

Figure 4: $\Delta Y$ versus row number for small beam.

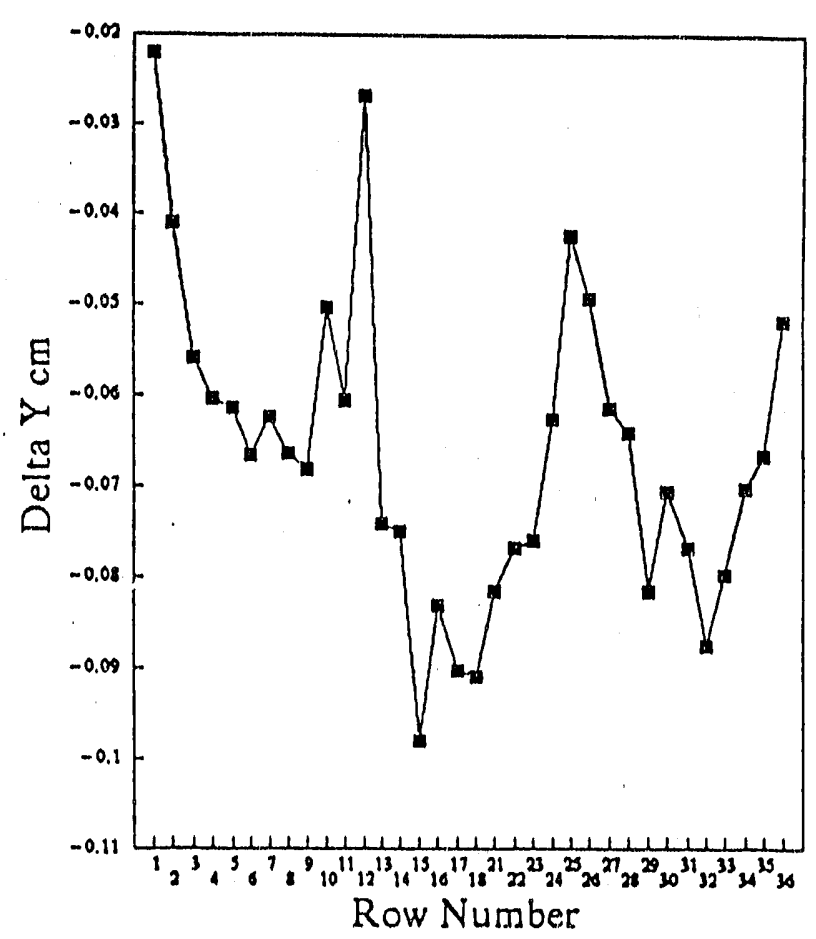

Figure 5: $\Delta Y$ versus row number for big beam.

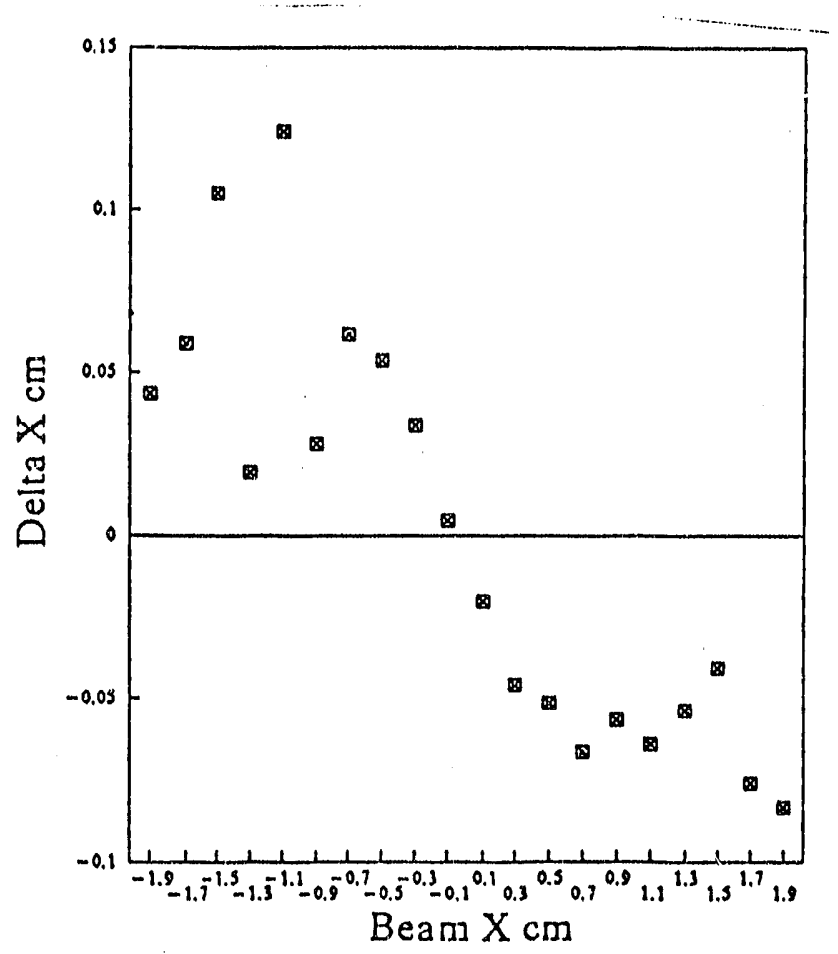

Figure 6: $\Delta X$ versus projected $X$ for small beam. 


\section{Two Vertex Resolution}

In experiment E-810 a typical event from $p-P b$ has 3 charged particles. After reconstruction, an event having a vertex displacement of 5 or more mm from the incoming beam position is easily distinguished. Therefore we expect at RHIC where $A u-A u$ collisions produce several thousand charged particles, events with even smaller two vertex separations can be distinguished. This is important because the TPC live time is $50 \mu \mathrm{s}$. We expect $p-p$ collisions to occur at $10^{6}$ per second; an average of 50 events would occur in the live time of the TPC. Only a few percent of these will produce an apparent overlap within the vertex resolving space of two events. This condition can be detected by other devices such as silicon vertex detectors and TOF counters. These occurrences can simply be rejected from the data sample. It should be noted that 50 overlapping, $p-p$ collisions produce fewer charged particles than a single $A u-A u$ collision. Even at the highest RHIC luminosity the only penalty one pays for this long live time is increased event processing time since all tracks must be reconstructed before out of time collision can be rejected. E- 810 has demonstrated that the processing time is proportional to the number of tracks since a TPC directly yields 3 dimensional points along the tracks.

\section{Reconstruction Efficiency At High Track Density}

In experiment E-810 the track density in the front of the first TPC is shown in Fig. 7. During this run the TPC's had only $2 / 3$ of the rows operating. Under these conditions the track reconstruction efficiency is greater than $90 \%$. In the "STAR" TPC the maximum track density is predicted to be less than $0.1 / \mathrm{cm}^{2}$ and therefore the reconstruction efficiency is expected to be in the high 90 's. It should be noted that in both cases the tracking is initiated at the low density (downstream end) of the TPC system but successfully proceeds into the high density region near the interaction.

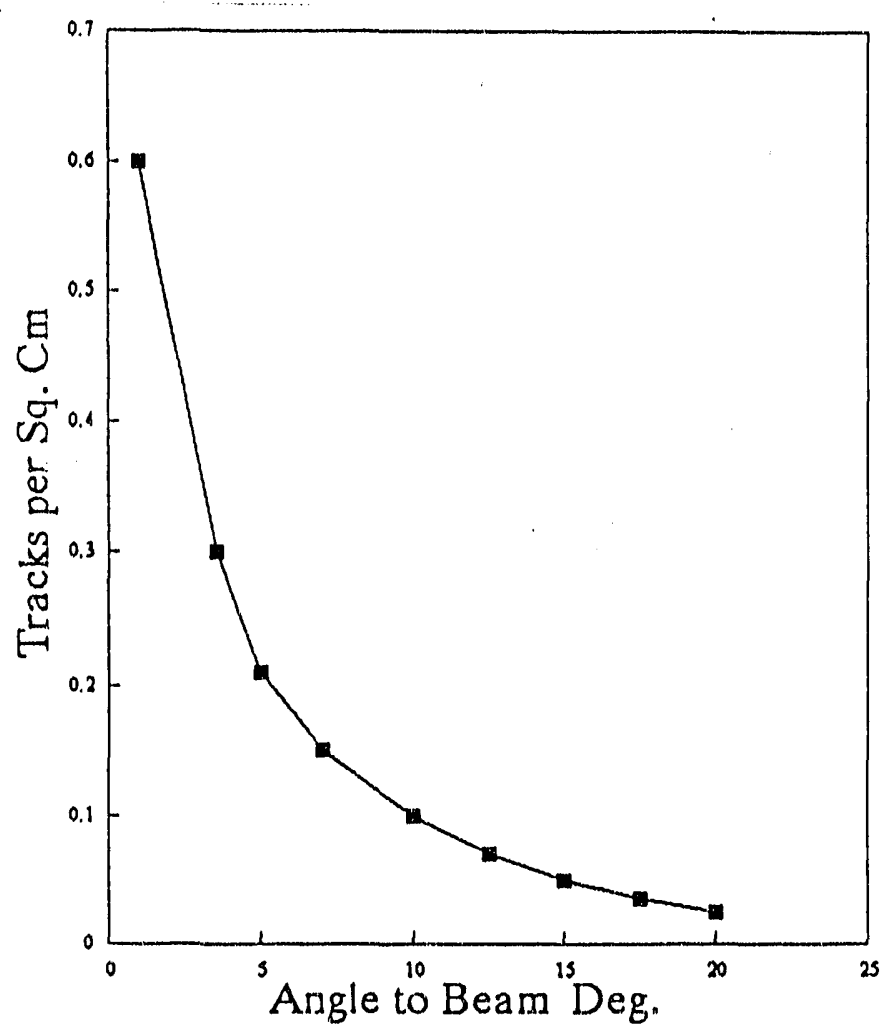

Figure 7: Track density in the first row of E-810 TPC's as a function of angle. 


\section{Robustness For Efficiency At High Particle Fluxes}

At track densities of $10^{7}$ minimum particles per second per $\mathrm{cm}^{2} \mathrm{PWC}$ 's and drift chambers lose gain because each track produces an avalanche of $\approx 10^{5}$ per primary ionization. Thus the ion loading in the gas is $10^{5}$ times higher than in a TPC where only tracks from events of interest are allowed to enter the avalanche space by a gating structure. In addition the gate prevents positive ions from the avalanche from entering the drift space. Therefore only the ions from the primary ionization can produce distortion in the electric field. Remarkably, this makes the TPC more robust to high event rates than either PWC's or drift chambers since they cannot be gated and there is no mechanism to trap the positive ions from the avalanche process. In the RHIC environment there can be large bursts of particles caused by distributed losses. In the TPC it will be possible in most cases to prevent the opening of the gate and therefore prevent excessive current in the TPC detector end caps.

\section{TPC Development for RHIC}

About half of the FY91 funding for R \& D was for travel between BNL and LBL that led to the merger of LOI\#3 and LOI\#5. The rest was spent on a pad readout test TPC and miscellaneous electronics. Most of the productive effort was the result of simulations on high rate, high track density performance of TPC's which were confirmed by measurements with the E- 810 apparatus, shown previously in Fig. 1.

Software was developed to simulate the electrostatic field. In addition electron drift in this field is simulated all the way to the charge distribution induced in the pad readout. One important result of this simulation is better that $200 \mu$ position resolution is achieved by recording charge on the 3 pads nearest the avalanche even with a $20 \%$ noise contribution.

Progress was also made in custom electronics for TPC readout, two parallel efforts are under way. The charge amplifier-shaper and analog memory efforts at LBL are discussed in another paper. The BNL effort consists of a transresistance amplifier-shaper under development at UCSC that has a very high degree of integration and reamarkably low power. We expect to have prototypes for testing early in 1992. An analog storage with data compaction logic IC is under development at Analytek under an SBIR contract. This is complementary to the LBL analog memory effort.

A prototype radial drift TPC is being designed and will be built for testing in calendar 1992 running at the MPS. 'This radial TPC is proposed for external tracking at "STAR".

\section{Conclusions}

Measurements of distortions due to ion loading in the E-810 TPC's have been found to be small even at very high effective particle fluxes. This result supports the use of TPC's at RHIC even at the highest planned luminosity. In addition measurements of two vertex resolution and reconstruction efficiency at high track density show that a TPC is an excellent detector for RHIC. 


\section{References}

[1] A. Etkin, et al. "Modular TPC's for Relativistic Heavy-Ion Experiments", Nuclear Instruments and Methods, Vol. A2837, No. 3, pp. 557-566, November 1989. A. Etkin, et al. "A TPC for Large Solid Angle Relativistic Heavy Ion Experiments", IEEE Transactions on Nuclear Science, Vol. 36, No. 1, Part I, pp. 58, February 1988.

[2] "STAR", Solenoidal 'Tracker At RHIC, approved major detector for RHIC, see LBL31040, 1991 and LBL-29651, 1990. K. Kadija, G. Paic, D. Vranic - Rudjer Boskovic Institute; G. Danby, S. Eiseman, A. Etkin, K.J. Foley, R.W. Hackenburg, M.J. LeVine, R.S. Longacre, W. A. Love, E.D. Platner, A.C. Saulys, J.H. Van Dijk - Brookhaven National Laboratory; F.P. Brady, J.E. Draper, J.L. Romero - University of California at Davis; J.B. Carroll, V. Ghazikhanian, E. Gulmez, T.J. Hallman, G.J. Igo, S. Trentalange, C.A. Whitten, Jr. - University of California at Los Angeles; M. Kaplan, P. Karol, Z. Milosevich, E. Vardaci - Carnegie-Melon University; M.G. Cherney - Creighton U. niversity; S. Margetis, R.E. Renfordt, D. Röehrich, R. Stock, H. Ströebele, S. Wenig - University of Frankfurt; L. Madansky - The Johns Hopkins University; B.D. Anderson, D. Keane, R. Madey, J. Watson - Kent State University; F. Bieser, M.A. Bloomer, D.A. Cebra, W. Christie, E. Friedlander, D. Greiner, C. Gruhn, J.W. Harris, H. Huang, P.M. Jacobs, S.A. Kleinfelder, P.J. Lindstrom, H. Matis, C. McParland, R. Morse, C.J. Naudet, G. Odyniec, D.L. Olson, A.M. Poskanzer, G. Rai, H.-G. Ritter, I. Sakrejda, J.O. Schambach, L.S. Schroeder, P.A. Seidl, T.M. Symons, H. Wieman, W.K. Wilson - Lawrence Berkeley Laboratory; C.S. Chan, M.A. Kramer, S.J. Lindenbaum - City College of New York; A. Aprahamian, N.N. Biswas, U. Garg, V.P. Kenney, J. Piekarz - University of Notre Dame; T. Humanic University of Pittsburgh; D.D. Carmony, Y. Choi, A. Hirsch, E. Hjort, N.T. Porile, R.P. Scharenberg, B. Srivastava, M.L. Tincknell - Purdue University; D.L. Adams, S. Ahmad, B.E. Bonner, J.A. Buchanan, C.N. Chiou, J.M. Clement, M.D. Corcoran, T. Empl, H.E. Miettinen, G.S. Mutchler, J.B. Roberts, J. Skeens, I. Stancu - Rice University; A.D. Chacon, K.L. Wolf - Texas A \& M University; W. Dominik, M. Gazdzicki - Warsaw University; T. Pawlak, W. Peryt, J. Pluta - Warsaw University of Technology; W.J. Braithwaite, J.G. Cramer, D. Prindle, T. Trainor, University of Washington; A. Breskin, R. Chechik, Z. Fraenkel, I. Tserruya - Weizmann Institute of Science. 


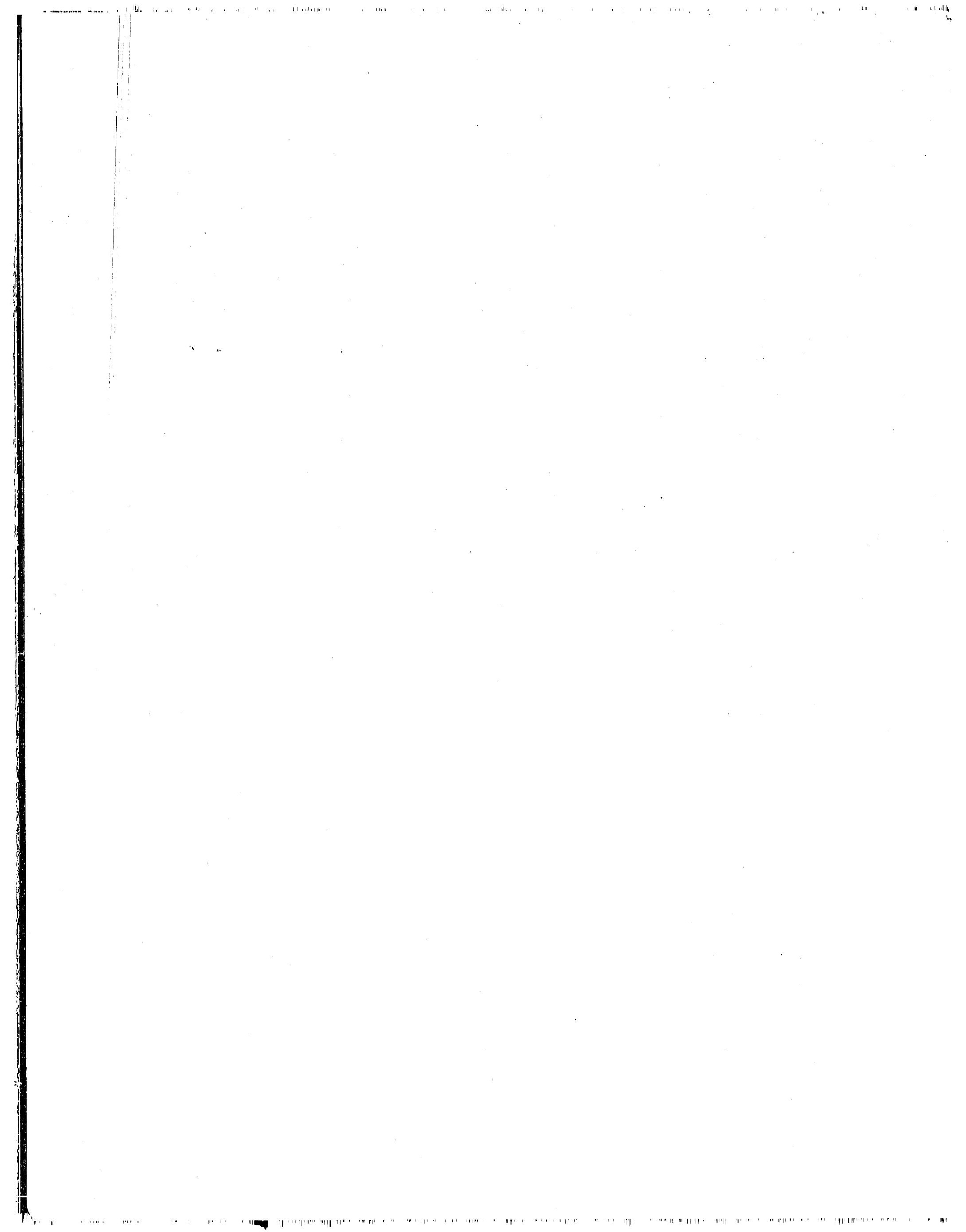

